1)

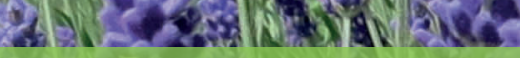

\title{
PLANTS' RESPONSES TO \\ NOVEL ENVIRONMENTAL \\ PRESSURES
}

\section{EDITED BY: Alessio Fini, Massimiliano Tattini and Raquel Esteban PUBLISHED IN: Frontiers in Plant Science}

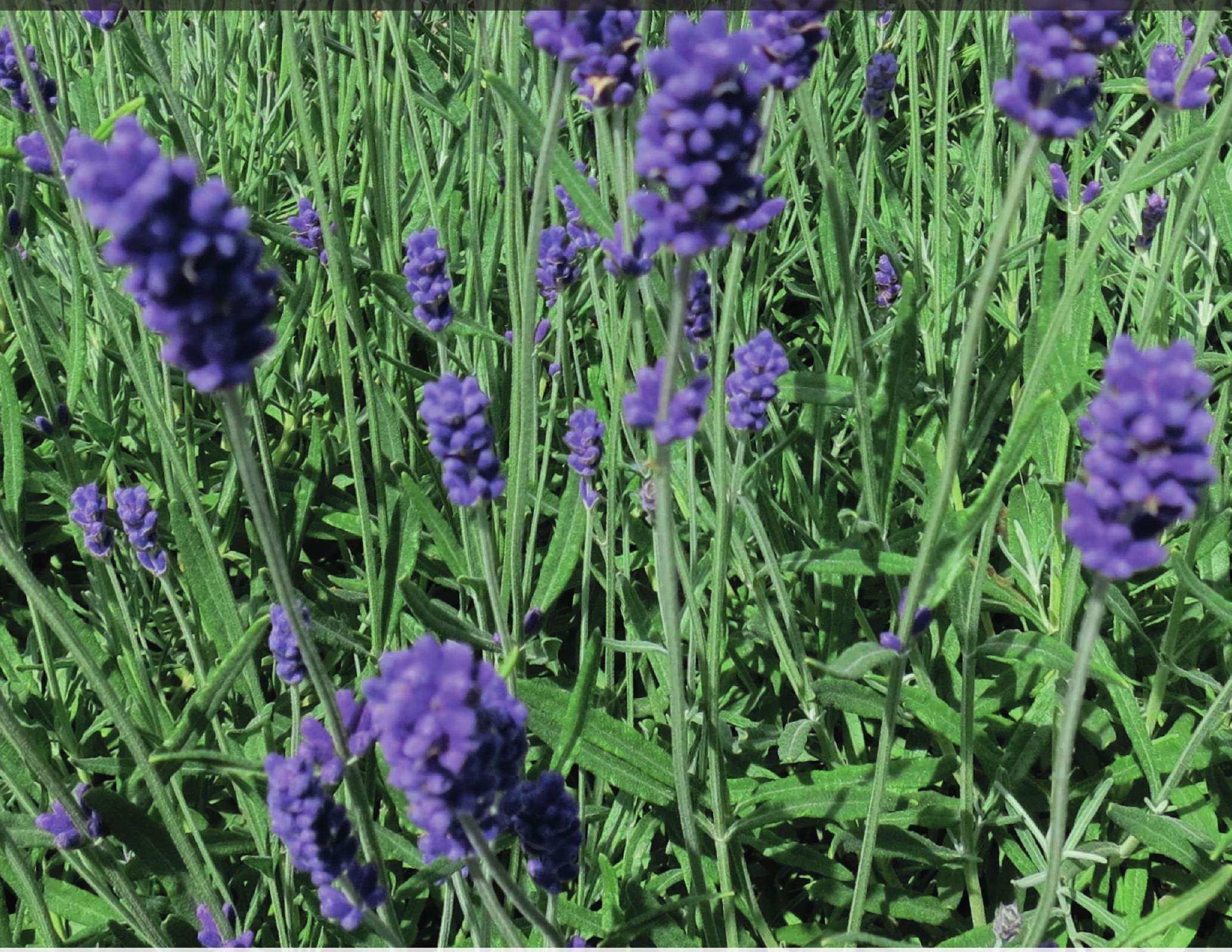




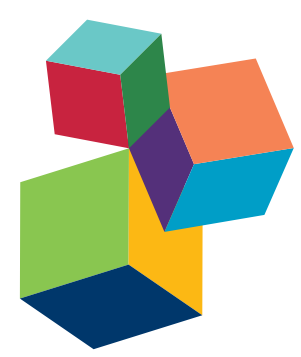

Frontiers Copyright Statement

(c) Copyright 2007-2018 Frontiers Media SA. All rights reserved.

All content included on this site, such as text, graphics, logos, button

icons, images, video/audio clips,

downloads, data compilations and

software, is the property of or is

licensed to Frontiers Media SA

("Frontiers") or its licensees and/or subcontractors. The copyright in the text of individual articles is the property of their respective authors, subject to a license granted to Frontiers.

The compilation of articles constituting

this e-book, wherever published,

as well as the compilation of all other

content on this site, is the exclusive property of Frontiers. For the conditions for downloading and copying of e-books from Frontiers' website, please see the Terms for Website Use. If purchasing Frontiers e-books from other websites or sources, the conditions of the website concerned apply.

Images and graphics not forming part of user-contributed materials may not be downloaded or copied without permission.

Individual articles may be downloaded and reproduced in accordance with the principles of the CC-BY licence subject to any copyright or other notices. They may not be re-sold as an e-book.

As author or other contributor you grant a CC-BY licence to others to reproduce your articles, including any graphics and third-party materials supplied by you, in accordance with the Conditions for Website Use and subject to any copyright notices which you include in connection with your articles and materials.

All copyright, and all rights therein, are protected by national and international copyright laws.

The above represents a summary only. For the full conditions see the Conditions for Authors and the Conditions for Website Use.

ISSN 1664-8714 ISBN 978-2-88945-402-0

DOI 10.3389/978-2-88945-402-0

\section{About Frontiers}

Frontiers is more than just an open-access publisher of scholarly articles: it is a pioneering approach to the world of academia, radically improving the way scholarly research is managed. The grand vision of Frontiers is a world where all people have an equal opportunity to seek, share and generate knowledge. Frontiers provides immediate and permanent online open access to all its publications, but this alone is not enough to realize our grand goals.

\section{Frontiers Journal Series}

The Frontiers Journal Series is a multi-tier and interdisciplinary set of open-access, online journals, promising a paradigm shift from the current review, selection and dissemination processes in academic publishing. All Frontiers journals are driven by researchers for researchers; therefore, they constitute a service to the scholarly community. At the same time, the Frontiers Journal Series operates on a revolutionary invention, the tiered publishing system, initially addressing specific communities of scholars, and gradually climbing up to broader public understanding, thus serving the interests of the lay society, too.

\section{Dedication to Quality}

Each Frontiers article is a landmark of the highest quality, thanks to genuinely collaborative interactions between authors and review editors, who include some of the world's best academicians. Research must be certified by peers before entering a stream of knowledge that may eventually reach the public - and shape society; therefore, Frontiers only applies the most rigorous and unbiased reviews.

Frontiers revolutionizes research publishing by freely delivering the most outstanding research, evaluated with no bias from both the academic and social point of view. By applying the most advanced information technologies, Frontiers is catapulting scholarly publishing into a new generation.

\section{What are Frontiers Research Topics?}

Frontiers Research Topics are very popular trademarks of the Frontiers Journals Series: they are collections of at least ten articles, all centered on a particular subject. With their unique mix of varied contributions from Original Research to Review Articles, Frontiers Research Topics unify the most influential researchers, the latest key findings and historical advances in a hot research area! Find out more on how to host your own Frontiers Research Topic or contribute to one as an author by contacting the Frontiers Editorial Office: researchtopics@frontiersin.org 


\section{PLANTS' RESPONSES TO NOVEL ENVIRONMENTAL PRESSURES}

Topic Editors:

Alessio Fini, University of Milan, Italy

Massimiliano Tattini, National Research Council of Italy (CNR), Institute for Sustainable Plant Protection, Italy

Raquel Esteban, University of the Basque Country (UPV/EHU), Spain

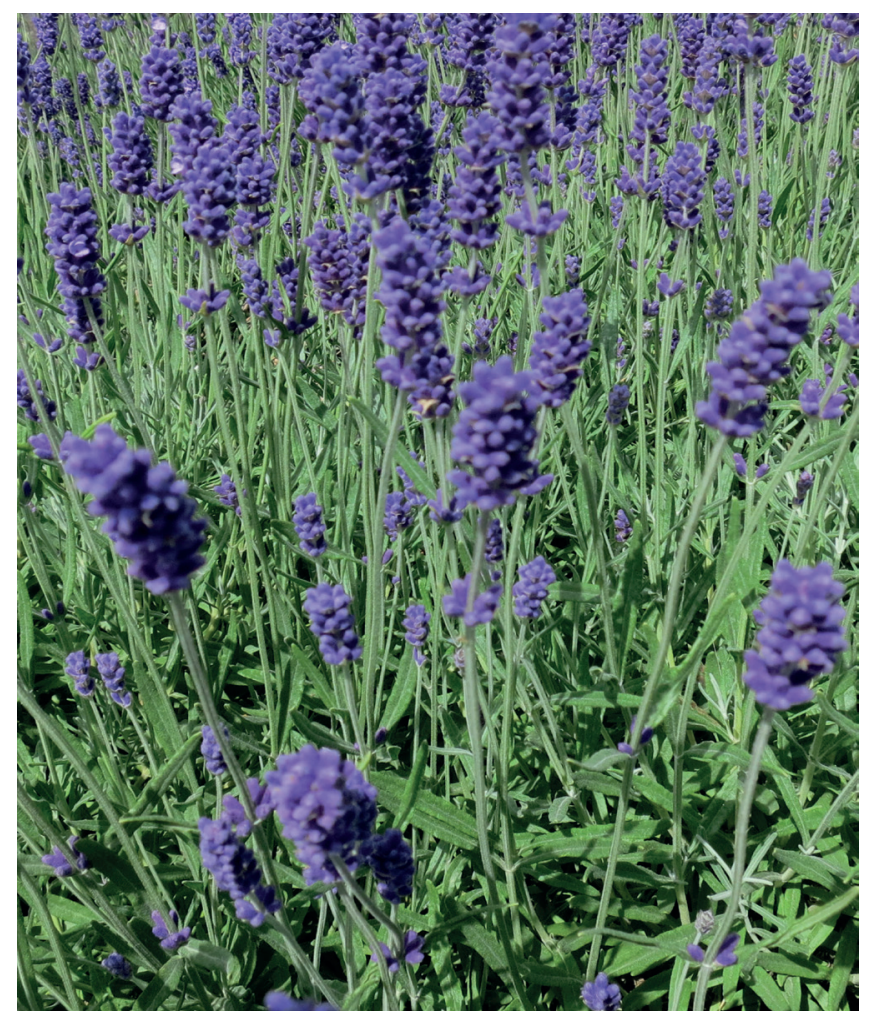

Levander, a native Mediterranean plant to the dry and heat Mediterranean regions, expanding northwards due to global change. Here, an urban planting in Copenhagen.

Image: Alessio Fini.

Plants have been exposed to multiple environmental stressors on long-term (seasonal) and short-term (daily) basis since their appearance on land. However, the frequency and the intensity of stress events have increased much during the last three decades because of climate change. 
Plants have developed, however, a multiplicity of modular and highly integrated strategies to cope with challenges imposed by novel, usually harsher environments. These strategies include migration, acclimation and adaptation. Twelve articles in this research topic exactly focus on the relative significance of these response mechanisms for the successful acclimation of plants to a wide range of novel environmental pressures. Four articles, additionally, explore how plants respond to severe stress conditions resulting from the concurrent action of multiple stressors. Ten articles mostly examine how morpho-anatomical, physiological and biochemical-related traits integrate when plants suffer from 'novel' threats, such as solid, gaseous, and electromagnetic pollutants. Suitable physiological indicators for developing conservation strategies are described in the last two works. This research topic highlights that bottom-up, as well as, topdown approaches will be necessary to develop in near future in the study of plants' responses to environmental pressures.

Citation: Fini, A., Tattini, M., Esteban, R., eds. (2018). Plants' Responses to Novel Environmental Pressures. Lausanne: Frontiers Media. doi: 10.3389/978-2-88945-402-0 


\section{Table of Contents}

07 Editorial: Plants' Responses to Novel Environmental Pressures

Alessio Fini, Massimiliano Tattini and Raquel Esteban

Chapter 1: Migration, Adaptation, and Acclimation - The

"Flight Strategy" of Plants

11 UV Screening in Native and Non-native Plant Species in the Tropical Alpine: Implications for Climate Change-Driven Migration of Species to Higher Elevations

Paul W. Barnes, Ronald J. Ryel and Stephan D. Flint

22 Potential Responses of Vascular Plants from the Pristine "Lost World" of the Neotropical Guayana Highlands to Global Warming: Review and New Perspectives

Valentí Rull and Teresa Vegas-Vilarrúbia

30 Effects of Environment and Space on Species Turnover of Woody Plants across Multiple Forest Dynamic Plots in East Asia

Yun Chen, Zhiliang Yuan, Peikun Li, Ruofan Cao, Hongru Jia and Yongzhong Ye

41 Phylogeny, Seed Trait, and Ecological Correlates of Seed Germination at the Community Level in a Degraded Sandy Grassland

Zhengning Wang, Lixin Wang, Zhimin Liu, Yanjuan Li, Qingqing Liu and Bo Liu

51 Habitat Temperature and Precipitation of Arabidopsis thaliana Ecotypes

Determine the Response of Foliar Vasculature, Photosynthesis, and

Transpiration to Growth Temperature

William W. Adams III, Jared J. Stewart, Christopher M. Cohu, Onno Muller and

Barbara Demmig-Adams

69 Photoprotective Strategies of Mediterranean Plants in Relation to Morphological Traits and Natural Environmental Pressure: A Meta-Analytical Approach

Beatriz Fernández-Marín, Antonio Hernández, Jose I. Garcia-Plazaola, Raquel Esteban, Fátima Míguez, Unai Artetxe and Maria T. Gómez-Sagasti

85 Dissecting Long-Term Adjustments of Photoprotective and Photo-Oxidative Stress Acclimation Occurring in Dynamic Light Environments

Shizue Matsubara, Trang Schneider and Veronica G. Maurino

90 Biogeochemical and Ecomorphological Niche Segregation of Mediterranean Woody Species along a Local Gradient

Enrique G. de la Riva, Teodoro Marañón, Cyrille Violle, Rafael Villar and

Ignacio M. Pérez-Ramos 
Chapter 2: The Significance of the "Flight Strategy" in the Anthropocene Era

99 Plant Physiological, Morphological and Yield-Related Responses to Night Temperature Changes across Different Species and Plant Functional Types Panpan Jing, Dan Wang, Chunwu Zhu and Jiquan Chen

118 Functional and Integrative Analysis of the Proteomic Profile of Radish Root under $\mathbf{P b}$ Exposure

Yan Wang, Liang Xu, Mingjia Tang, Haiyan Jiang, Wei Chen, Wei Zhang, Ronghua Wang and Liwang Liu

132 Alternative Growth and Defensive Strategies Reveal Potential and Gender Specific Trade-Offs in Dioecious Plants Salix paraplesia to Nutrient Availability Hao Jiang, Sheng Zhang, Yanbao Lei, Gang Xu and Dan Zhang

141 Seasonal, Sex- and Plant Size-Related Effects on Photoinhibition and Photoprotection in the Dioecious Mediterranean Dwarf Palm, Chamaerops humilis

Melanie Morales, Marta Pintó-Marijuan and Sergi Munné-Bosch

Chapter 3: How Plants Respond to Multiple Stressors

153 Climate Influences the Content and Chemical Composition of Foliar Tannins in Green and Senesced Tissues of Quercus rubra

Sara M. Top, Caroline M. Preston, Jeffrey S. Dukes and Nishanth Tharayil

165 Infestation of Broad Bean (Nicia faba) by the Green Stink Bug (Nezara viridula) Decreases Shoot Abscisic Acid Contents under Well-Watered and Drought Conditions

Luisa Ederli, Cecilia Brunetti, Mauro Centritto, Stefano Colazza, Francesca Frati, Francesco Loreto, Giovanni Marino, Gianandrea Salerno and Stefania Pasqualini

177 Interactive Effects of UV-B Light with Abiotic Factors on Plant Growth and Chemistry, and Their Consequences for Defense against Arthropod Herbivores Rocio Escobar-Bravo, Peter G. L. Klinkhamer and Kirsten A. Leiss

191 Abiotic Stress Responses and Microbe-Mediated Mitigation in Plants: The Omics Strategies

Kamlesh K. Meena, Ajay M. Sorty, Utkarsh M. Bitla, Khushboo Choudhary, Priyanka Gupta, Ashwani Pareek, Dhananjaya P. Singh, Ratna Prabha, Pramod K. Sahu, Vijai K. Gupta, Harikesh B. Singh, Kishor K. Krishanani and Paramjit S. Minhas

Chapter 4: Solid, Gaseous, and Electromagnetic Pollution: A Novel Oxidative Threat to Plants

216 Linking Changes to Intraspecific Trait Diversity to Community Functional Diversity and Biomass in Response to Snow and Nitrogen Addition Within an Inner Mongolian Grassland Wei Mao, Andrew J. Felton and Tonghui Zhang

226 Losing the Warning Signal: Drought Compromises the Cross-Talk of Signaling Molecules in Quercus ilex Exposed to Ozone

Lorenzo Cotrozzi, Elisa Pellegrini, Lucia Guidi, Marco Landi, Giacomo Lorenzini, Rossano Massai, Damiano Remorini, Mariagrazia Tonelli, Alice Trivellini, Paolo Vernieri and Cristina Nali

239 Isoprene Responses and Functions in Plants Challenged by Environmental Pressures Associated to Climate Change

Alessio Fini, Cecilia Brunetti, Francesco Loreto, Mauro Centritto, Francesco Ferrini and Massimiliano Tattini 
247 Contrasting Hydraulic Architectures of Scots Pine and Sessile Oak at Their Southernmost Distribution Limits

Elisabet Martínez-Sancho, Isabel Dorado-Liñán, Uwe G. Hacke, Hannes Seidel and Annette Menzel

259 Moderate Drought Stress Induces Increased Foliar Dimethylsulphoniopropionate (DMSP) Concentration and Isoprene Emission in Two Contrasting Ecotypes of Arundo donax

Matthew Haworth, Stefano Catola, Giovanni Marino, Cecilia Brunetti, Marco Michelozzi, Ezio Riggi, Giovanni Avola, Salvatore L. Cosentino, Francesco Loreto and Mauro Centritto

270 Timing Effects of Heat-Stress on Plant Ecophysiological Characteristics and Growth

Dan Wang, Scott A. Heckathorn, Kumar Mainali and Rajan Tripathee

281 Differential Response of Floating and Submerged Leaves of Longleaf Pondweed to Silver lons

Nisha Shabnam, P. Sharmila, Govindjee, Hyunook Kim and P. Pardha-Saradhi

295 Nanoparticles Alter Secondary Metabolism in Plants via ROS Burst Gregory Marslin, Caroline J. Sheeba and Gregory Franklin

303 A Streamlined Approach by a Combination of Bioindication and Geostatistical Methods for Assessing Air Contaminants and Their Effects on Human Health in Industrialized Areas: A Case Study in Southern Brazil

Angélica B. Ferreira, Andreza P. Ribeiro, Maurício L. Ferreira, Cláudia T. Kniess, Cristiano C. Quaresma, Raffaele Lafortezza, José O. Santos, Mitiko Saiki and Paulo H. Saldiva

318 Electromagnetic Field Seems to Not Influence Transcription via CTCT Motif in Three Plant Promoters

Dariusz Sztafrowski, Anna Aksamit-Stachurska, Kamil Kostyn, Paweł Mackiewicz and Marcin Łukaszewicz

Chapter 5: Plants Under Global Change: Physiological Indicators and Monitoring Tools

332 Observing Climate Change Impacts on European Forests: What Works and What Does Not in Ongoing Long-Term Monitoring Networks

Filippo Bussotti and Martina Pollastrini

337 Drought Tolerance in Pinus halepensis Seed Sources As Identified by Distinctive Physiological and Molecular Markers

Khaled Taïbi, Antonio D. del Campo, Alberto Vilagrosa, José M. Bellés,

María Pilar López-Gresa, Davinia Pla, Juan J. Calvete, José M. López-Nicolás and José M. Mulet 